\title{
The Coalescence Instability in Solar Flares
}

T.Tajima and F.Brunel, University of Texas; J.-I.Sakai, Toyama University and University of Maryland; L.Vlahos and M.R.Kundu, University of Maryland

\begin{abstract}
The non-linear coalescence instability of current carrying solar loops can explain many of the characteristics of the solar flares such as their impulsive nature, heating and high energy particle acceleration, amplitude oscillations of electromagnetic emission as well as the characteristics of 2-D microwave images obtained during a flare. The plasma compressibility leads to the explosive phase of loop coalescence and its overshoot results in amplitude oscillations in temperatures by adiabatic compression and decompression. We note that the presence of strong electric fields and super-Alfvenic flows during the course of the instabilty paly an important role in the production of non-thermal particles. A qualitative explanation on the physical processes taking place during the non-linear stages of the instability is given.
\end{abstract}

\section{Introduction}

Direct observations in soft x-rays (Howard and Svestka, 1977) of interconnecting coronal loops suggest that loop coalescence may be a very important process for energy release in the solar corona. It was suggested (Tajima, Brunel and Sakai, 1982) that the most likely instability for impulsive energy release in solar flares is the coalescence instability (Tajima 1982; Brunel, Tajima and Dawson, 1982). In the present article we examine the existing observational and theoretical results together with a global energy transfer model and conclude that the merging of two current carrying solar loops can explain many of the known characteritics of solar flares.

\section{Observations}

In this section we briefly outline several recent observational results, which agree well with the model of two interacting loops which will be presnted in the following section.

The hard $\mathrm{x}$-ray emission from solar flares results from the interaction of energetic electrons with protons and ions, with electron energies ranging from 10 to hundred keV's in the upper part of the chromosphere (Brown, 1971). The microwave emission, on the other hand, is interpreted as gyro-synchrotron emission resulting from the gyration of the energetic electrons around the magnetic field.

The microwave emission is closely correlated with hard x-ray emission (Kundu, 1961). With the availability of SMM and Hinotori experiments and ground based observations using the Very Large Array (VLA), it has been possible to obtain spatially resolved two-dimensional images of the microwave burst sources and of hard $\mathrm{x}$-ray sources with energies less than $30 \mathrm{keV}$. The main conclusions that emerge from the analysis of the existing observations are that the microwave emission is often confined on the upper part of a closed magnetic loop (Marsh and Hurford, 1980; Kundu et al., 1982) and hard x-ray emission is mainly emitted from the foot points of the loop 
T.Tajima, F.Brunel, J.Sakai, L.Vlahos, M.R.Kundu

The Coalescence Instability in Solar Flares

(Duijvemant et al., 1982; Hoyng et al., 1983). The time structure of the impulsive emission is usually "spiky" and is characterized by pulses of short duration (Kiplinger et al., 1983; Kaufmann et al., 1983).

The two-dimensional maps which were obtained with the VLA during an impulsive flare observation May 14, 1980 probably provide the first direct evidence of a coalescence of coronal loops (Kundu et al., 1982).

The $6 \mathrm{~cm}$ burst appeared as a gradual component on which was superimposed a strong impulsive phase (duration $\sim 2$ minutes) in coincidence with a hard x-ray burst. Soft $\mathrm{x}$-ray emission (1.6 $\sim 25 \mathrm{keV}$ ) was associated with the gradual burst (before the impulsive burst), as is to be expected. There is a delay of hard $\mathrm{x}$-ray emission $(>28 \mathrm{keV})$ relative to $6 \mathrm{~cm}$ emission $(\sim 10 \mathrm{sec}$ delay from $6 \mathrm{~cm}$ max to $\mathrm{x}$-ray start and $\sim 20 \mathrm{sec}$ delay from $6 \mathrm{~cm} \max$ to $\mathrm{x}$-ray max.). The preflare region showed intense emission with peak $T_{b} \sim 10^{7} \mathrm{k}$ extended along a neutral line situated approximately in the east-west direction. A burst source of intense emission with $T_{b} \sim 4 \times 10^{7} \mathrm{k}$, appeared initially. The most remarkable feature of the burst source evolution was that an intense emission extending along the north-south neutral line (line of zero polarization at $6 \mathrm{~cm}$ ), possibly due to reconnection, appeared just before the impulsive burst occurred. This northsouth neutral line must be indicative of the appearance of a new system of loops. In the 20 seconds preceding the impulsive peak $\left(\mathrm{T}_{\mathrm{b}} \sim 1.1 \times 10^{9} \mathrm{~K}\right)$ the arcade of loops (burst source) changed and ultimately developed into two strong bipolar regions or a quadrupole structure whose orientations were such that near the loop tops the field lines were opposed to each other. The impulsive energy release must have occurred due to magnetic reconnection of the field lines connecting the two oppositely polarized bipolar regions.

Observations of gamma-rays and high-energy neutrons are a relatively new and useful diagnostic of relativistic particle acceleration in solar flares. Gamma-ray lines are the products of nuclear reaction between flare accelerated protons and nuclei with the ambient solar atmosphere. Narrow line emission results from the de-excitation of nuclear levels in solar atmospheric nuclei, such as $\mathrm{C}, \mathrm{O}, \mathrm{Ne}, \mathrm{Mg}$, $\mathrm{Si}$ and $\mathrm{Fe}$, and from neutron capture and positron annihilation. Broad-band nuclear emission results from the superposition of such lines and deexcitation radiation from excited heavy nuclei in the energetic particle population. Of particular significance is the 4-7 MeV band in which the nuclear lines $\mathrm{C}, \mathrm{N}$ and $\mathrm{O}$ produce the bulk of the observed emission. The strongest narrow line from disk flares in the $2.223 \mathrm{MeV}$ line results from neutron capture on $\mathrm{H}$ in the photosphere. The underlying gamma-ray continuum in solar flares is produced by bremsstrahlung from relativistic electrons.

Gamma-ray lines and continuum are formed from the interaction of $\mathrm{MeV}$ electrons and $\mathrm{GeV}$ ions in the low chromosphere or upper photosphere (average plasma density $\simeq 10^{14} \mathrm{~cm}^{-3}$ ). An interesting result that can be explained with the present flare model is the appearance of a "double peak" in the amplitude profile of the June 7 and 21, 1980 observation of gamma-ray emission (Forrest et al., 1981; Nakajima et al., 1982). These oscillations are present in the electron and ion temperature profiles on the numerical simulation presented below and can be explained from the dynamics of the coalescence instability. 


\section{The coalescence instability}

It is well known that the annihilation of magnetic energy and its conversion into kinetic energy by the tearing instability (Furth et al., 1963) are too slow to account for the impulsive energy release in solar flares. Many authors (Sweet, 1958; Parker, 1963; Petschek, 1964) have proposed fast magnetic reconnection mechanisms. Recently Tajima (1982) found that the reconnection rate for a compressible plasma with weak toroidal magnetic field $B_{t}$ is much larger (by a factor of $10^{2} \sim 10^{3}$ ) than that for a nearly incompressible plasma with large $B_{t}$ and that the sharp transition in reconnection behavior takes place when the poloidal field $B_{p}$ (created by the field aligned current $\mathrm{J}_{t}$ ) exceeds approximately $\mathrm{B}_{t}$. Brunel et al. (1982) found further that when the plasma is compressible a faster second phase of reconnection sets in after one Alfven time of the Sweet-Parker first phase with reconnected flux

$$
\psi=\psi_{\mathrm{sp}}\left(\mathrm{t}_{\mathrm{A}}\right) \cdot\left(\mathrm{t} / \mathrm{t}_{\mathrm{A}}\right)^{\rho_{\mathrm{i}} / \rho_{e}},
$$

where $\psi_{\text {sp }}$ is the Sweet-Parker flux

$$
\psi_{\mathrm{sp}}(\mathrm{t})=\eta^{1 / 2} \mathrm{~B}_{\mathrm{p}}(\mathrm{y}=\mathrm{a})\left(\rho_{1} / \rho_{\mathrm{e}}\right)^{1 / 2}\left(\mathrm{v}_{\mathrm{A} \perp} \mathrm{L}\right)^{1 / 2} \mathrm{t} \text {, }
$$

$\rho_{\mathrm{i}}$ and $\rho_{\mathrm{e}}$ are densities inside and outside of the current channel $\left(\rho_{\mathrm{i}} \geqq \rho_{\mathrm{e}}\right), \mathrm{t}_{\mathrm{A}}$ the Alfven time $\left(\mathrm{t}_{\mathrm{A}}=\right.$ $\left.\mathrm{a} / \mathrm{v}_{\mathrm{A} \perp}=\mathrm{a}(4 \pi \rho)^{1 / 2} / \mathrm{B}\right), \eta$ the resistivity, a the current channel width, and $\mathrm{L}$ the length of reconnecting region. According to the above theory, the annihilation of the magnetic flux proceeds much faster $\left(\psi \infty \mathrm{t}^{\rho_{i} / \rho_{\mathrm{e}}}\right)$ than the Sweet-Parker rate $(\psi \propto \mathrm{t})$, where for compressible plasmas $\rho_{\mathrm{i}} / \rho_{\mathrm{e}}>1$. When the plasma has a strong toroidal field and the plasma is incompressible, the reconnection rate reduces to the Sweet-Parker rate even for $t>t_{A}$. The theory is in good agreement with the computer simulation results of Brunel et al., 1982 and Tajima, 1982. Nevertheless the reconnection process (before the coalescence instbility starts) in itself, however fast it is, is not responsible for the large magnetic energy conversion into particle energy, but rather the change is in the magnetic geometry before and after the reconnection process. Indeed only a small fraction of the total poloidal magnetic energy is released through the reconnection process which necessarily takes place at the $\mathrm{x}$-point, i.e. the field null point, where not much magnetic energy is available in the first place.

It is the non-linear development of the coalescence instability of the current filaments (loops) that can release a large amount of magnetic energy (Wu et al., 1981; Leboeuf et al., 1982). Although the coalescence instability is of ideal magnetohydrodynamic (MHD) nature in the linear stage and the growth rate for compressible plasmas is somewhat smaller than that for incompressible cases (Pritchett et al., 1979), the non-linear development of this instability involves field line reconnection and therefore is of nonideal MHD nature. Since the reconnection rate drastically differs by two or three orders of magnitude (Tajima, 1982), the non-linear coalescence time differs by two or three orders of magnitude for case $B_{p} \gtrsim B_{t}$ and case $B_{p} \leqq B_{t}$. In fact the recent study (Bhattacharjee et al 1983) confirmed that the rapid coalescence occurs in a compressible plasma as characterized by Eq. (1). We studied the coalescence of two current filaments in detail using analytical and computer simulation techniques. 


\section{a. The simulation model}

A plasma configuration which is unstable against the tearing and subsequent coalescence instabilities has been studied by a fully selfconsistent electromagnetic relativistic particle simulation code (Langdon et al., 1976; Lin et al., 1974).

\section{b. Short description of time sequence}

When the linear stage where the energy release is small is past and two magnetic islands (i. e. current filaments) approach, the islands are squashed. The plasma near the contact area of islands is squeezed and has a high density, which leads to fast reconnection according to Eq. (1). Because of this, the total flux reconnection of two islands into a coalesced island takes place only within 1 2 Alfven times according to the simulation of Tajima et al., 1982. The magnetic energy contained in the island fields is explosively released into ion and electron kinetic energy as seen in Fig. 1. The ion temperature shown in Fig. 1 sharply increases over the non-linear coalescence stage in 1 2 Alfven times. Significantly, there appear amplitude oscillations in the electron and ion temperature. This temperature oscillation behavior can be attributed to the overshooting of coalescing and colliding two current blobs.

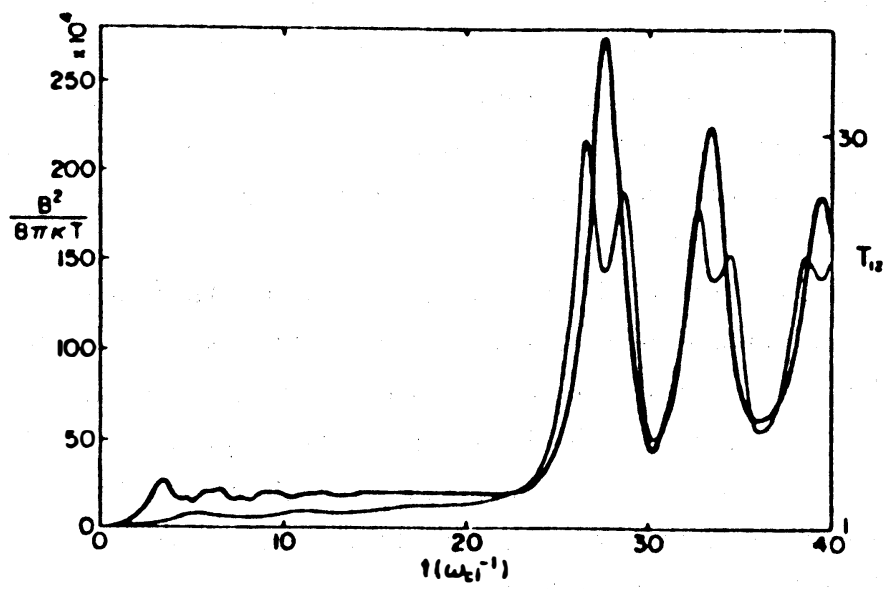

Fig. 1 Magnetic field energy and ion temperature oscillations before and after the coalescences of the current loops.

\section{c. Velocity distributions-heating}

Once two current blobs coalesce, they are bound by the common magnetic flux and the coalesced larger island which also vibrates, with a smaller amplitude. Within the coalesced island the colliding two plasma blobs cause turbulent flows which dissipate their energy quickly into heat, thereby reducing the amplitude of the temperature oscillations. As a result, the momentum distribution of plasma electrons and ions [shown in Fig. 2(b) in Tajima et al., 1982] exhibit the intense bulk heating and acceleration of the tail. The heating in the poloidal direction 
is due to adiabatic compression and decompression of the coalesced loops. The eventual bulk heating is a result of turbulent dissipation of counter streaming instabilities (either the Buneman instability or the modified two stream instability). The temperature in the poloidal direction was increased in our simulation by a factor of 60 . The heating in the toroidal direction is due to heating/acceleration by the inductive toroidal electric field which is several times the classical Dreicer field. The momentum distribution of ions in the toroidal direction shows three regimes, the first being the bulk, the second a exponential section, and the third a flat distribution up to the relativistic regime the relativistic factor $\gamma \sim 2$, where $\mathrm{p}_{o}{ }^{2} / 2 \mathrm{M}=10 \times$ (bulk temperature). The momentum distribution of electrons in the toroidal direction shows two regimes, the first being the bulk, the second a flat distribution up to the relativistic region.

\section{d. Temperature pulsations-double peaks}

The double peaks in the time development of the temperature (Fig. 1) occur just before $(t=$ $\left.t_{1} \sim 27 \omega_{c i}^{-1}\right)$ and after $\left(t=t_{3} \sim 29 \omega_{c i}^{-1}\right)$ the maxima of magnetic fields $\left(t=t_{2}\right)$. In Fig. 2 schematic sequential pictures of plasma dynamical behaviour during coalescence are shown. At $t=t_{1}$, the magnetic $(j \times B)$ acceleration of ions becomes maximum so that the magnetic flux behind the colliding plasma blobs as well as plasma blobs is strongly compressed. This plasma compression causes the first temperature peak at $t=t_{1}$. After this maximum acceleration phase ions acquire super-Alvenic velocities along the direction in which the loops are collide so that they detach from the magnetic flux against which ions have been compressed. This results in an expansion phase $\left(t=t_{2}\right)$ of ions and hence in an adiabatic cooling of the plasma as the magnetic fields obtain maximum values. The process reverses after the maxima of the magnetic fields at $t=t_{3}$ $\sim 29 \omega^{-1} \mathrm{c} i$, which gives rise to the second peak of the temperature.

\section{e. High energy tail acceleration-electrostatic fields}

The high energy tail particle acceleration of ions and electrons is probably due to a combination of localized electrostatic field acceleration across the poloidal magnetic field (Sagdeev and Shapiro, 1973 Sugihara and Midzuno, 1979, Sakai and Sugihara, 1983) and magnetic acceleration of the poloidal to toroidal directions. As shown in Fig. 2(a), electrons are magnetized and are carried away with the magnetic flux, while ordinary ions are accelerated by the $\mathrm{J} \times \mathrm{B}$ force. On the other hand the high energy ions are dragged by the charge separation created near the piled flux.

Ions (as well as electrons) acquire super-Alfvenic velocities upon coalescence. The difference of motions between ions and electrons around $t=t_{1}$ causes a strong localized shock-like electrostatic field, $E$, which propagates with a velocity $V_{p}$. In Fig. 3, the density distribution and shock-like electrostatic fields just before the coalescence $\left(t=t_{1}\right)$ are shown. This $V_{p} \times B$ acceleration causes the formation of high energy particles in the toroidal direction. By this acceleration process, ions and electrons are accelerated to relativistic energies in opposite directions along the toroidal magnetic field.

The temperature anisotropy between the poloidal and toroidal component of the velocity distribution that was observed in our simulation may give rise to the onset of the Alfven-ion cyclotron instability (Tajima et al., 1980). This instability may create large amplitude Alfven 
T.Tajima, F.Brunel, J.Sakai, L.Vlahos, M.R.Kundu

The Coalescence Instability in Solar Flares

(a) $t=t_{1}$

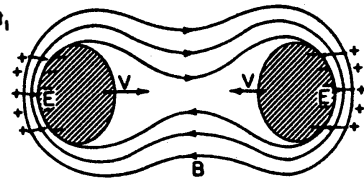

(b) $1=1_{2}$

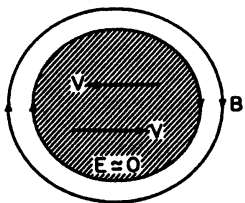

(c) $1=t_{3}$

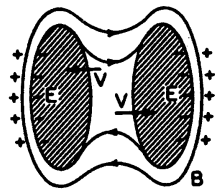

Fig. 2 Schematic sequential of pictures of the plasma dynamics during the coalescence.

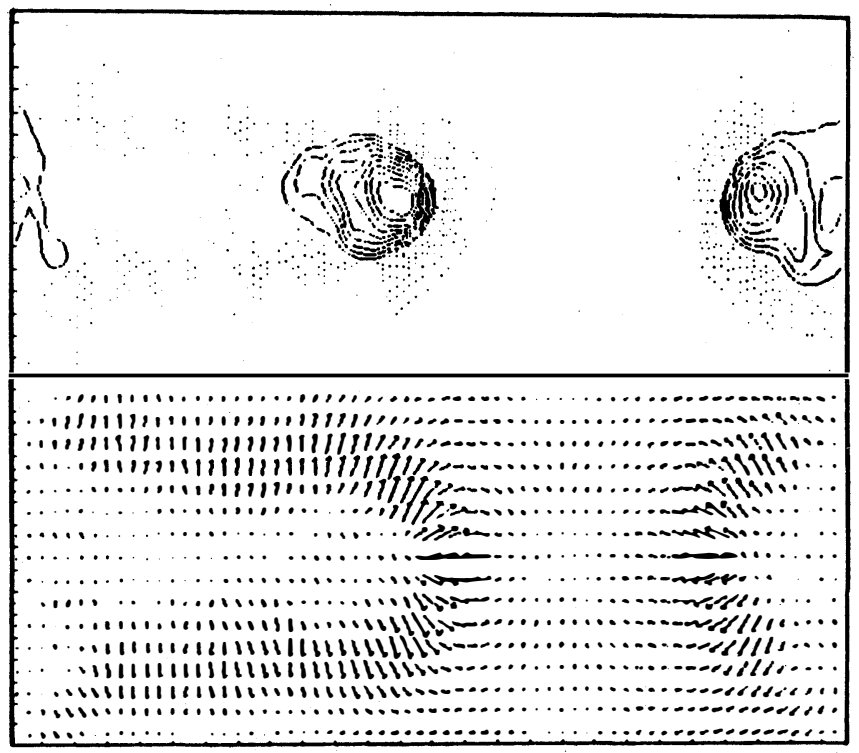

Fig. 3 The density and electrostatic field distribution just before the coalescence.

waves that are traveling away from the coalescence region as well as ion cyclotron resonance heating by Alfven waves with frequencies near the cyclotron frequency.

\section{f. The role of $B_{p} / B_{t}$ on the growth}

Our simulations show that the non-linear coalescence time for case $B_{p}>B_{t}$ is more rapid by two or three orders of magnitude than the case $B_{p} \leq B_{t}$. When the toroidal magnetic field is 
stronger than the poloidal field, the super-Alfvenic plasma flows cause a plasma rotational motion around the toroidal magnetic field, rather than the counter streaming flow. The result of plasma incompressibility leads to weaken the double peak structure in the temperature oscillations.

\section{g. Flare energetics}

The flare loop slowly expands after it emerges from the photosphere as the toroidal field curvature of the loop makes the centrifugal motion. However, its relative stable configuration suggests that the quiet loop is in some kind of equilibrium such as the Waltier-Taylor equilibrium which is a force-free stable. In time the toroidal current $\mathrm{J}_{t}$ builds up increasing the poloidal magnetic field $B_{p}$. As the poloidal field $B_{p}$ reaches a critical value which is of the order of $B_{t}$ in magnitude, the adjacent flare current loop can coalesce rapidly, being facilitated by the fast reconnection process governed by Eq.(1) (the faster second phase). A process similar to this that can happen upon increased toroidal current $\mathrm{J}_{\mathrm{t}}$ (or more twisted magnetic tube) is the (global) kink instability. This instability creates a local section of parallel currents, which may also coalesce rapidly. Such a fast coalescence of flare current loops proceeds explosively once in its non-linear regime in a matter of 1-2 Alfven times, releasing more that $1 / 10$ of the poloidal magnetic energy into (ion) kinetic energy. Since the flare loop magnetic field $(100 \mathrm{G})$ with current rod size $\left(\mathrm{a}=10^{8}\right.$ $\mathrm{cm}), \mathrm{W}_{\mathrm{c}} \sim 0.5 \times 10^{20} \mathrm{ln}(\mathrm{L} / \mathrm{a}) \sim 1.5 \times 10^{20} \mathrm{erg} / \mathrm{cm}$ and the energy available in length $\mathrm{d} \sim \mathrm{L}\left(\sim 10^{9} \mathrm{~cm}\right)$ is $\mathrm{E}=1.5 \times 10^{29} \mathrm{erg}$ for $\mathrm{a}=10^{8} \mathrm{~cm}, \mathrm{~d}=\mathrm{L}=10^{9} \mathrm{~cm}$ and $\mathrm{E}=1.5 \times 10^{31} \mathrm{erg}$ for $\mathrm{a}=10^{9} \mathrm{~cm}, \mathrm{~d}=\mathrm{L}=10^{10} \mathrm{~cm}$. Release of ion energy, therefore, is $\mathrm{E}_{\mathrm{ion}} \sim \frac{1}{6} \mathrm{E}$ is in between $2 \times 10^{28} \mathrm{erg}$ and $2 \times 10^{30} \mathrm{erg}$ due to the coalescence. This amount of energy is in the neighborhood of the energy released in a solar flare (Sturrock, 1980). For magnetic field $\simeq 100 \mathrm{G}$ the Alfven time is of the order of $1 \mathrm{sec}$., which is approximately the time scale for the explosive coalescence. The time scale of the impulsive phase is observed to be several seconds, and is in good agreement with the above theoretical estimate. The sudden nature of the impulsive flare phase (Sturrock, 1980) is thus explained by increasing field aligned current and the faster second phase reconnection in the course of coalescence.

\section{Radiation signatures from the coalescence of two solar loops}

In order to relate the observations with the physical picture of the coalescence instability reported above one must analyze the energy transfer in a 3-D (global) magnetic topology that connects the interacting loops and follow the evolution of the energetic particles and the hot plasma away from the energy release volume. In Figure 4 we present the magnetic topology of the interacting loops during the coalescence. As was pointed out in the previous section the byproducts of the interaction are: (a) the generation of hot electron and ion distributions; (b) the existence of run-away tails on the ion and electron distribuitons, and (c) the presence of MHD and electrostatic waves emitted away from the energy release volume. In what follows we will summarize how each of these components will evolve in time and locate the most likely place for the emission of the microwave, $\mathrm{x}$-ray, $\gamma$-ray and meter wave-length bursts associated with the coalescence instability. 
T.Tajima, F.Brunel, J.Sakai, L.Vlahos, M.R.Kundu

The Coalescence Instability in Solar Flares

a. Energetic electrons and ions stream away from the energy release region following the magnetic field lines. The majority of these electrons and ions will reach the chromosphere in a fraction of a second. The interaction of these particles with the chromosphere will result in bremsstrahlung hard x-ray, gamma-ray continuum and gamma-ray lines. A fraction of the high energy electron population will be trapped in the upper part of the interacting loops and emit gyro-synchrotron radiation. We expect that if the VLA resolves these structures we will observe two sources with two different neutral lines. Each radio source will be split into two oppositely polarized sources. This picture is indeed in good agreement with observations; in particular the May 14 event (Kundu et al., 1982). Energetic electrons and ions can also be accelerated by the

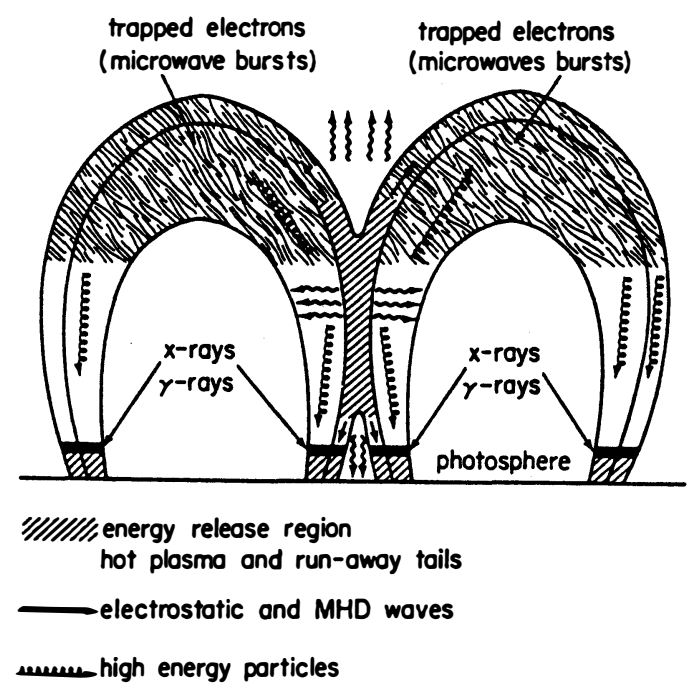

Fig. 4 A schematic model for the energy transfer and location of different radiation signatures that results during the coalescence of two solar. loops.

electrostatic waves excited from the unstable currents inside the energy release volume. Electrostatic waves propagating away from their excitation region will be absorbed in different places inside the merged loops. For example if the drift velocity of the currents during coalescence exceeds the ion speed, the energy release region becomes a source of lower hybrid waves which propagate across the magnetic field with phase velocity along the field lines $\geq 3 \mathrm{v}_{\mathrm{e}}$ can easily accelerate electrons up to $100 \mathrm{keV}$ energies (Tanaka and Papadopoulos, 1983). These waves will progressively propagate further towards the center of the merged loops since accelerated electrons escape away from the interaction region and locally reduce the damping. The net result is that a relatively large volume around the energy release region can be affected by the electrostatic waves. Excitation of electrostatic ion-cyclotron waves will have the same effect on the ions. We thus suggest that the nonthermal tails co-existing with the hot plasma inside the energy release region and the waves driven by the unstable currents inside it will be responsible for the prompt acceleration of the trapped and precipitated population of energetic electrons and ions that will radiate the microwave, hard $\mathrm{x}$-ray and gamma-ray emission. 
b. The hot (several keV) electron and ion component will expand along the field lines forming heat waves propagating along the field lines. It is possible that under certain conditions (see Brown, Melrose and Spicer, 1979 or Vlahos and Papadopoulos, 1979) the heat conduction will be anomalously inhibited due to the formation of ion acoustic fronts in the interface of the hot plasma with the surrounding material, at the same time the chromosphere will also be heated by the precipitated electrons and ions. The result is another hydromagnetic heat wave expanding in the opposite direction. This expansion phase lasts only a few seconds and unless one of the interacting loops is larger $\mathrm{L} \gtrsim 10^{10} \mathrm{~cm}$, it will not be observed with the presently available instru ments. The mixture of the hot coronal plasma with the dense and hot chromospheric plasma will be responsible for the appearance of two post-flare loops that will slowly cool off during the decay phase of the flare.

c. MHD waves: Finally a significant amount of energy is emitted in the form of hydromagnetic waves which may or may not steepen to form shocks. These waves are traveling away from the interaction region with velocity $\left(v_{s}\right)$ larger than the local Alfven speed $\left(v_{A}\right)$.

They can fill a volume $\mathrm{V}\left(\mathrm{V} \simeq\left(\mathrm{V}_{\mathrm{s}} \tau\right)^{3}\right.$ which can be as large as $\left.10^{27} \mathrm{~cm}^{3}\right)$ in a few seconds. The interaction of these waves with the ambient plasma and the pre-accelerated electrons will increase the number and average energy of the energetic particle population. Most of these electrons and ions will end up in the chromosphere but a fraction of them will escape in the interplanetary space.

Although in the discussion above we qualitatively analyze the coalescence of two isolated loops, one must keep in mind that in most cases many such collisions take place simultaneously or nearly simultaneously, lasting somctimes several minutes. In these cases the MHD waves excited from each individual collision join together to form a large volume with turbulent wave activity that accelerate clectrons and ions to very high energies with good efficiency. The hydromagnetic waves excited from the coalescence instability can destabilize another solar loop (Sakai and Washimi, 1982, Sakai, 1983, Sakai, Tajima and Brunel, 1984) which, as it expands, forms a coronal transient (Sakai 1982) and a shock that drives type II and/or type IV bursts.

\section{Summary}

The hot plasma, non-thermal particles and waves (electrostatic and MHD) which are present during the coalescence instability can explain several of the observed characteristics of solar flares. The interaction of solar loops in the manner described here are quite common in the sun but due to the fact that only if $B_{p} / B_{t}$ is small the coalescence instability is rapid, not all of them produce impulsive, energetic flares.

\section{Acknowledgements}

This work was supported at the University of Texas by NSF grant ATM82-14730 and DOE grant DE-FGO5-80 ET53088, and at the University of Maryland by NASA grant NAG W-81 and NSF grant 81-03089. One of the authors (J.Sakai) would like to thank Drs.M.R.Kundu and T. 
T.Tajima, F.Brunel, J.Sakai, L.Vlahos, M.R.Kundu

The Coalescence Instability in Solar Flares

Tajima for their hospitality while visiting the University of Maryland and the Unversity of Texas.

\section{References}

Bhattacharjee, A., Brunel, F. and Tajima, T.: 1983, IFSR \#93, The University of Texas, Austin. Phys. Fluids 26, 3332.

Brown, J.C., Melrose, D.B. and Spicer, D.S.: 1979, Ap. J., 228, 592.

Brunel, F., Leboeuf, J.N., Tajima, T., Dawson, J.M., Makino, M. and Kamimura, T.: 1981, J. Comput. Phys. 43, 268.

Brunel, F., Tajima, T. and Dawson, J.M.: 1982, Phys. Rev. Lett., 49. 323.

Chupp, E.L., Forrest, D.J. and Suri, A.N.: 1974, in Solar Gamma-, X-, and EUV Radiation (edited by S. Kane) (Reidel, Holland) p. 341.

Duijvemont, A.P., Hoyng, P. and Machado, M.E.: 1982, Solar Physics, 81, 137.

Forrest, D.J., Chupp, E.L., Rayan, J.M., Reppin, C., Rieger, E., Kanbach, G., Pinkau, K., Share, G., and Kinzer, G.: 1981, to be published in the Late Volumes of the 17th International Cosmic Ray Conference; Paris, France.

Furth, H.P., Killeen, J. and Rosenbluth, M.N.: 1963, Phys. Fluids, 6, 459.

Gold, T. and Hoyle, F.: 1960, Monthly Notices Roy. Astron. Soc., 120, 8.

Howard, R., Svestaka, Z.: 1977, Solar Phys., 54, 65.

Hoyng, P., Marsh, K.A., Zirin, H. and Denis, R.B.: 1983, Ap. J., 285, 865.

Kaufman et al.: 1983, Solar Physics, (in press).

Kiplinger, A.L., Denis, B.R., Emslie, A.G., Frost. K.J. and Orwig, L.E.: 1983, Solar Physics, (in press).

Kundu, M.R.: 1961, J. Geophys. Res., 66, 4308.

Kundu, M.R., Schmahl, E.J., Velusamy, T. and Vlahos, L.: 1982, Astron. Astrophys.,108, 188.

Nakajima, H., Kosugi, T., Kai, K., and Enome, S.: 1983, Nature, (in press).

Langdon, A.B. and Lasinski, B.F.: 1976, Methods in Computational Physics, Academic, New York, Vol. 16, p. 327.

Leboeuf, J.N., Tajima, T. and Dawson, J.M.: 1982, Phys. Fluids, 25, 784.

Lin, A.T., Dawson, J.M. and Okuda, H.: 1974, Phys. Fluids, 17, 1995.

Marsh, K.A. and Hurford, G.T.: 1980, Ap. J., (Lett.), 240, 6111.

Parker. E.N.: 1963, Ap. J.Suppl. Ser., 77, 177.

Petschek, H.E.: 1964, in Symposium on the Physics of Solar Flares, ed. by W.H. Hess, NASA, Washington, p. 425.

Pritchett, P.L. and Wu, C.C.: 1979, Phys. Fluids, 22, 440.

Sagdeev, R.Z. and Shapiro, V.D.: 1973, JETP Lett., 17, 279.

Sakai, J.: 1982, Ap. J., 263, 970.

Sakai, J.: 1983, J. Plasma Phys. 30, 109.

Sakai, J. and Sugihara, R.: 1984 Proc. of IAU Symp. No. 107, College Park, Maryland U.S.A.(Aug 8-11,1983)

Sakai, J., Tajima, T. and Brunel, F.: 1984, Solar Physics (in press)

Sakai, J. and Washimi, H.: 1982, Ap.J., 258, 823. 
Sturrock, P.A.ed.: 1980, Solar Flares: Monograph from Skylab Solar Working II, Colorado Associated University Press, Boulder.

Sugihara, R. and Midzuno, Y.: 1979, J.Phys. Soc. Japan, 47, 1290.

Svestka, Z.: 1976, Solar Flares, Reidel, Holland.

Svestka, Z. and Howard, R.: 1981, Solar Phys., 71, 349.

Sweet, P.A.: 1958, Nuovo Cimento Suppl. 8 (Ser. 10), 188.

Tajima, T.: 1982, in Fusion Energy-1981, (Internatinal atomic Energy Agency, ICTP, Trieste, 1982) p. 403. Also IFSR\#4...

Tajima, T., Brunel, F. and Sakai, J.: 1982, Ap. J., 258, L45.

Tajima, T. and Dawson, J.M.: 1980, Nuclear Fusion, 20, 1129.

Tanaka, M. and Papadopoulos, K.: 1983, Phys. Fluids., 26, 1697.

Vlahos, L. and Papadopoulos, K.: 1979, Ap. J., 233, 717.

Wu, C.C., Leboeuf, J,N., Tajima, T. and Dawson, J.M.: 1983, Phys.

Fluids, submitted for publication.

This paper was presented on International Astronomical Union Symposium No.107 "Unstable

Current Systems and Plasma Instabilities in Astrophysics" held at College Park, Maryland, U.S.

A. (August 8-11, 1983) 